\title{
UNEXPECTED HEIGHT OFFSETS IN TANDEM-X: EXPLANATION AND CORRECTION
}

\author{
Gerhard Krieger, Francesco De Zan, Markus Bachmann, \\ Jaime Hueso Gonzalez, Marc Rodriguez Cassola, Manfred Zink
}

\author{
Microwaves and Radar Institute, German Aerospace Center (DLR), Oberpfaffenhofen, Germany
}

\begin{abstract}
This paper reports on systematic height offsets that have been observed in TanDEM-X by evaluating a large number of digital elevation model (DEM) acquisitions. Besides the expected instrument and baseline offsets, which are compensated in the calibration chain, two unexpected external error sources have been identified that apply to any formation flying bistatic SAR interferometer. The first contribution is due to relativistic effects and can be well explained within Einstein's special theory of relativity. The second effect is due to differential delays in the troposphere. It is shown that the theoretic predictions are in good agreement with the observed offsets. All necessary corrections have in the meantime been integrated in the operational TanDEM-X processing chain.
\end{abstract}

Index Terms: Synthetic Aperture Radar (SAR), Interferometry, Bistatic Synchronization, Relativity, Reference Frames, Tropospheric Delay

\section{INTRODUCTION}

The successful launch of the TanDEM-X mission in June 2010 opened a new era in radar remote sensing. TanDEM-X is the first bistatic formation-flying SAR interferometer in space and has the challenging goal to acquire a global DEM with unprecedented accuracy [1]. A key component to achieve this objective is the calibration of the bistatic SAR interferometer. This calibration is based on the systematic evaluation of a large number of interferometric TanDEM-X data takes. During this evaluation, some unexpected height offsets have been observed in both the interferometrically and the radargrammetrically derived DEMs. A part of these offsets could successfully be explained by internal instrument delays [2], but there remained some puzzling height offsets in the order of $10 \mathrm{~m}$ that varied systematically along the orbit and, to a smaller degree, also with the incident angle. This paper shows that the latitude dependent offsets can be explained by relativistic effects, while differential delays in the troposphere account for residual height variations that vary with the incident angle.

\section{RELATIVISTIC EFFECS}

\subsection{Reference Frames}

Global DEM acquisition with TanDEM-X is based on the bistatic mode, where one satellite is selected as transmitter and both satellites record simultaneously the scattered radar echoes from the ground. A prerequisite for this mode is the accurate synchronization between the SAR receivers. This is achieved in TanDEM-X via a bidirectional synchronization link that periodically exchanges radar pulses between the two SAR instruments using a pair of dedicated horn antennas. From this, a common time and phase reference can be established with high accuracy by an appropriate interpolation between the synchronization pulses. The comparison of repeated TanDEM-X data takes from the same test site verified this approach and demonstrated a synchronization accuracy in the order of $1^{\circ}$ [3]. A tacit assumption is, however, that the clock synchronization is performed in a reference frame that is linked to the transmitter and receiver platforms as illustrated in Figure 1.

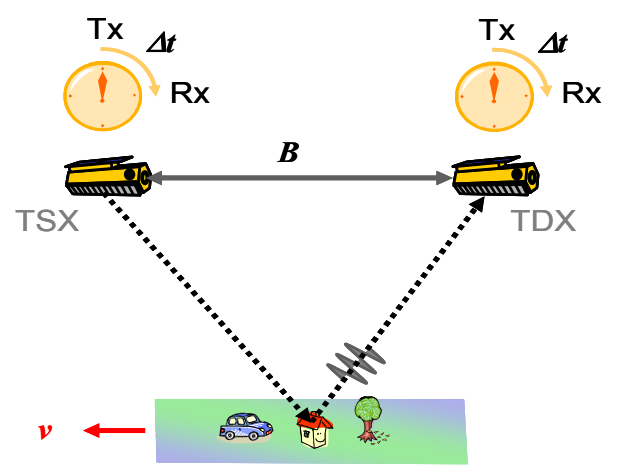

Figure 1: Bistatic SAR data acquisition as seen from a platform centered reference frame. The satellites are stationary while the scene moves as indicated by the red arrow. The $T x$ and $R x$ clocks are assumed to be perfectly synchronized in this reference frame.

In contrast, SAR focusing and interferometric DEM generation are performed in an Earth-Centered Earth-Fixed (ECEF) reference frame, where the two satellites move relative to a stationary target on the ground. This situation is illustrated in Figure 2.

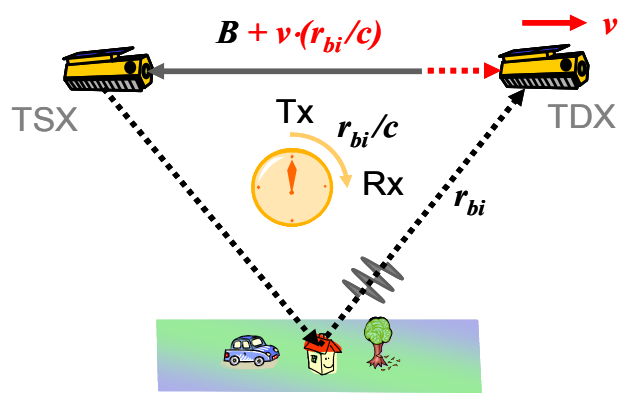

Figure 2: Bistatic SAR data acquisition as seen from an Earth Centred Earth Fixed (ECEF) reference frame where the scene is stationary and the satellites move. 


\subsection{Relativity of Simultaneity}

In 1905, Albert Einstein founded his special theory of relativity [4]. This theory postulates that the speed of light $c$ is an invariant and has always the same constant value, independent of the inertial reference frame that is used to describe a physical system. An immediate consequence of this postulate is the so-called non-simultaneity of events. This means that two spatially separated events, which happen at the same time in one reference frame, may no longer be simultaneous within another reference frame that moves relative to the first one. This is illustrated in Figure 3. As a result, radar transmitters and receivers, which operate perfectly synchronous in the platform centered frame, are no longer synchronous in the ECEF frame.
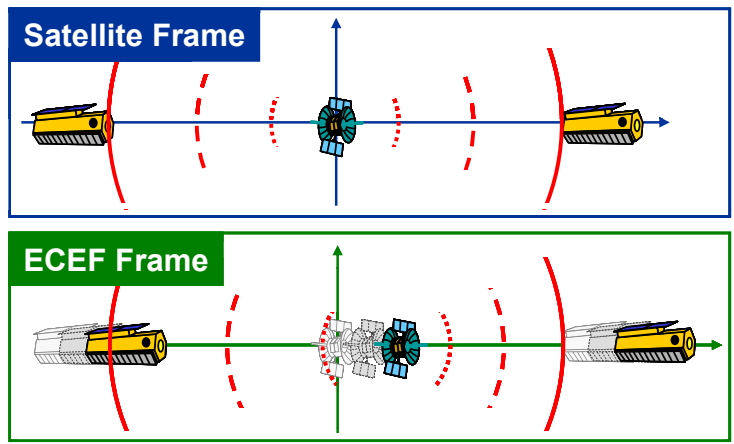

Figure 3: Illustration of non-simultaneity of events. A virtual satellite between TDX and TSX transmits pulses (red) which arrive at the same time (i.e. simultaneously) in the platform frame (top) but at different times (i.e. non-simultaneously) in the ECEF frame (bottom).

\subsection{Invariance of the Spacetime Interval}

A central concept in the theory of relativity is the invariance of the spacetime interval that can (in case of flat spacetime geometry) be written as [5]

$$
\Delta s^{2}=(c \cdot \Delta t)^{2}-\sum_{i=1}^{3} \Delta x_{i}^{2}
$$

where $\Delta t$ and $\Delta x_{i}$ denote, respectively, the time and position differences between two events as observed in a given reference frame ${ }^{1}$. From Figure 1 it becomes clear that, in the platform reference frame, the spacetime interval $\Delta s^{2}$ between the transmit (Tx) and receive ( $\mathrm{Rx})$ events is given by

$$
\Delta s^{2}=(c \cdot \Delta t)^{2}-\|\vec{B}\|^{2}
$$

where $\vec{B}$ is the baseline vector pointing from the transmitter satellite to the receiver satellite and $\Delta t$ is the elapsed time between the transmit and receive events. Using on the other hand the ECEF frame shown in Figure 2 to describe the same situation in a different coordinate system, the interval between the Tx and Rx events is provided by

\footnotetext{
${ }^{1}$ For those who are more familiar with the Lorentz transformations from special relativity, it is easy to show that $\Delta s$ remains invariant under the Lorentz group of linear spacetime transformations.
}

$$
\Delta s^{2}=\left(c \cdot \frac{r_{b i}}{c}\right)^{2}-\left\|\vec{B}+\vec{v} \frac{r_{b i}}{c}\right\|^{2}
$$

where $r_{b i}$ is the bistatic range and $\vec{v}$ is the velocity vector of the receiving satellite. Equating (2) and (3) yields

$$
(c \cdot \Delta t)^{2}-\|\vec{B}\|^{2}=\left(c \cdot \frac{r_{b i}}{c}\right)^{2}-\left\|\vec{B}+\vec{v} \frac{r_{b i}}{c}\right\|^{2}
$$

and after resolving for $r_{b i}$ one obtains:

$$
r_{b i}=\frac{2 \vec{B} \frac{\vec{v}}{c} \pm \sqrt{\left(2 \vec{B} \frac{\vec{v}}{c}\right)^{2}+4\left(1-\left(\frac{\|\vec{v}\|}{c}\right)^{2}\right)(c \cdot \Delta t)^{2}}}{2\left(1-\left(\frac{\|\vec{v}\|}{c}\right)^{2}\right)}
$$

For platform velocities that are small if compared to the speed of light, Equation (5) can be well approximated by

$$
r_{b i} \approx c \cdot \Delta t+\vec{B} \cdot \frac{\vec{v}}{c}
$$

The right hand side of this approximation is composed of two terms. The first term represents the product of the velocity of light with the time difference between the Tx and Rx events as measured in the platform system, where data acquisition and recording have been performed. A user unaware of relativistic effects would mistake this first term as a direct measure of the bistatic range in the ECEF frame. Taking into account the spacetime structure of special relativity, the second term emerges. This term is proportional to the scalar product between the platform velocity vector $\vec{v}$ and the baseline vector $\vec{B}$, i.e. it increases with both the along-track baseline between the satellites and the satellite velocity, i.e. the velocity difference between the ECEF and the platform frame.

\subsection{Relativity in TanDEM-X}

To investigate the relativistic effects in TanDEM-X, we note that the two satellites fly in a close Helix formation [1]. The Helix formation provides not only suitable cross-track baselines for the global DEM acquisition, but it is also characterized by a periodic variation of the along-track separation $B_{\text {along }}$ between the two satellites. For the present context, $B_{\text {along }}$ is approximated with sufficient accuracy by

$$
B_{\text {along }}(\Phi) \approx A \cdot \cos (\Phi)
$$

where $\Phi$ is the argument of latitude and $A$ is a constant that depends on the eccentricity offset between the two satellite orbits. Depending on the selected Helix formation, $A$ has typically values between $500 \mathrm{~m}$ and $900 \mathrm{~m}$.

Figure 4 shows the predicted relativistic range offsets for TanDEM-X as a function of latitude for $A=600 \mathrm{~m}$. Note that the sign of the shift flips by interchanging the role of the transmitter and receiver satellites. This dependency is evident from both Equation (6) and Figure 2. It can be seen that the magnitude of the bistatic range error varies within 
an interval of $\pm 15 \mathrm{~mm}$. While such an error may be considered small for bistatic localization and image registration, it will cause severe offsets in case of bistatic DEM generation. Such a DEM can be generated either radargrammetrically or interferometrically by combining the monostatic SAR image from the fully active transmitter with the bistatic SAR image acquired by the passive receiver. Neglecting the relativistic effect for the monostatic image, a range difference of $\pm 15 \mathrm{~mm}$ would translate to a height error of $\pm 24 \mathrm{~m}$ for a TanDEM-X acquisition with a height of ambiguity of $50 \mathrm{~m}$. From this, it becomes clear that relativistic effects cannot be neglected in the operational DEM generation chain of TanDEM-X.

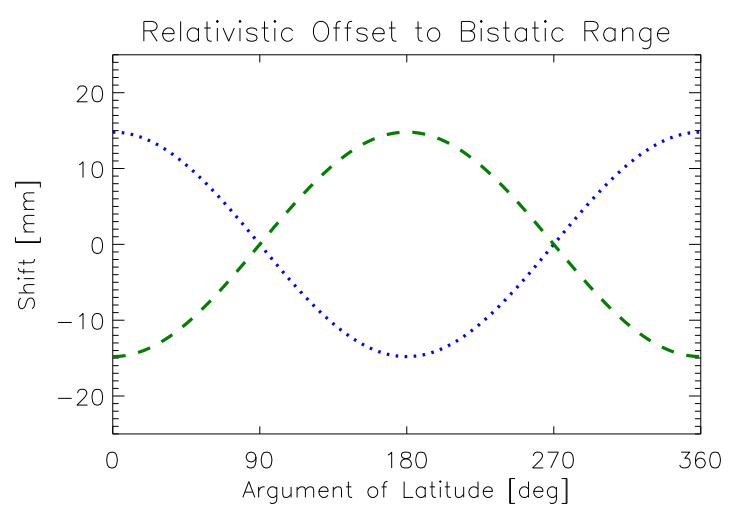

Figure 4: Predicted relativistic range offset for a typical TanDEM-X Helix formation with a maximum radial displacement of $300 \mathrm{~m}$ and a resulting variation of the alongtrack baseline between $\pm 600 \mathrm{~m}$. The dashed green curve shows the relativistic offsets if TSX is selected for transmission, while the dotted blue curve shows the predicted offsets in case that TDX is transmitting.

Figure 5 shows the radargrammetric offsets obtained with an early version of the TanDEM-X processor that did not take into account the relativistic effects. The offsets have been obtained by comparing TanDEM-X radargrammetric DEMs with external reference DEMs. Both the magnitude and the characteristic change of sign fit well with the prediction from Figure 4. Note that the figure contains also some other (smaller) instrument related calibration errors that have in the meantime been corrected [2].

Taking into account the relativistic correction and additional calibration steps in the operational TanDEM-X processor, the accuracy of the radargrammetric shifts is now typically below $\pm 5 \mathrm{~mm}$ where inaccuracies of the reference DEMs may be the dominant error source.

Figure 6 shows an even more clear dependency which can be explained by relativistic effects. The red and green crosses denote interferometric phase offsets that have been obtained by comparing interferometric TanDEM-X DEMs with reference DEMs ${ }^{2}$. Besides the $\pi$-ambiguity ${ }^{3}$, which is

\footnotetext{
${ }^{2}$ Note that the baseline is always computed from the transmitter (master) satellite, so that the sign flip is not visible in this figure.
}

resolved in the processor by radargrammetry, again a clear dependency on the along-track baseline can be seen. By comparing the measured data with the relativistic prediction, which is shown by the dashed blue lines, an excellent agreement is obtained. It becomes again clear that relativistic corrections are required to avoid systematic, latitude dependent offsets in the final DEMs. Note that without the relativistic correction the interferometric phase values would be almost randomly cluttered among a complete ambiguity interval. This caused in the beginning a significant confusion within the TanDEM-X engineering team.



Figure 5: Measured radargrammetric shifts as a function of latitude. The shifts were obtained by comparing TanDEM-X radargrammetric DEMs to reference DEMs (this plot is from the commissioning phase and contains also some other (smaller) errors that have been corrected in the meantime [2]).

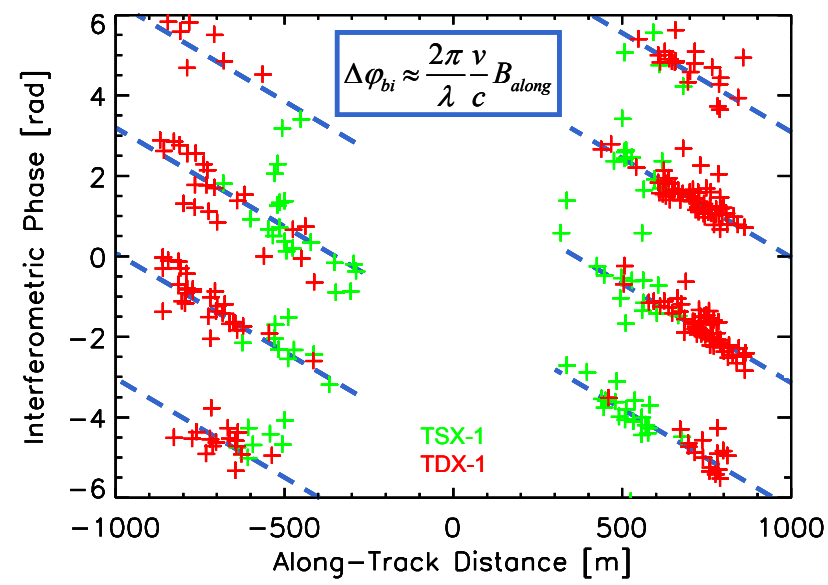

Figure 6: TanDEM-X interferometric phase offsets as a function of the along-track distance between the satellites. The relativistic prediction (dashed blue lines) agrees well with the measurements (crosses).

\footnotetext{
${ }^{3}$ The $\pi$-ambiguity is a consequence of the bi-directional synchronization technique where the average of two phase values is
} evaluated. 


\section{DIFFERENTIAL TROPOSPHERIC DELAYS}

The evaluation of a large number of TanDEM-X DEMs after implementation of the relativistic corrections revealed another systematic height offset that increases with increasing incident angles. Figure 7 shows this offset as a function of the beam number where \#1 corresponds to the near range beam with an incident angle of $\sim 30^{\circ}$ and $\# 9$ to far range with an incident angle of $48^{\circ}$. It becomes clear that the offset between near and far range is in the order of $2 \mathrm{~m}$.

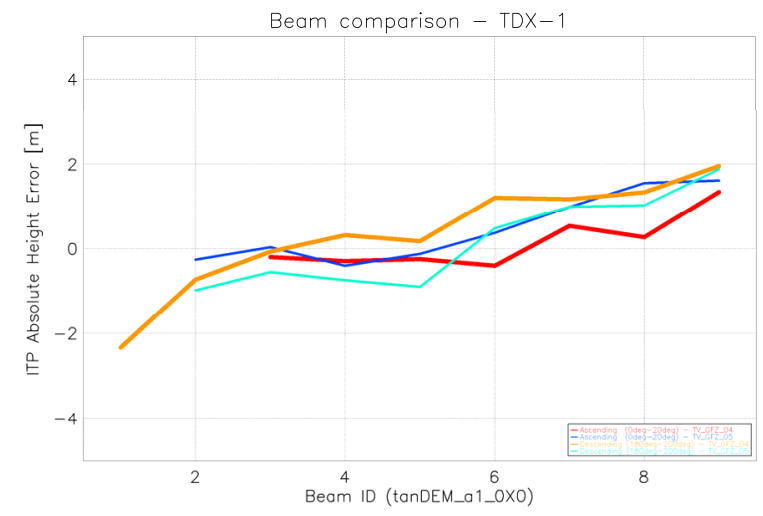

Figure 7: Height offsets as a function of beam number (\#1 and $\# 9$ correspond to incident angles of $30^{\circ}$ and $48^{\circ}$, respectively). The different curves refer to ascending and descending orbits as well as to different baseline product revisions.

Figure 8 shows that these systematic offsets can be explained by differential tropospheric delays. For this, one should be aware that the two satellites see the ground from slightly different incident angles. The radar echoes scattered from the ground travel hence somewhat different distances through the troposphere which results in turn in a difference in the tropospheric signal delay. Let's furthermore assume that the troposphere is, within a sufficient spatial neighborhood, horizontally homogeneous and characterized by a constant zenith delay $x_{\text {zenith }}$. It is then straightforward to compute the differential delay $\Delta r$ as illustrated on the right hand side of Figure 8 . The predicted height offsets are shown in Figure 9. Note the good agreement with Figure 7.

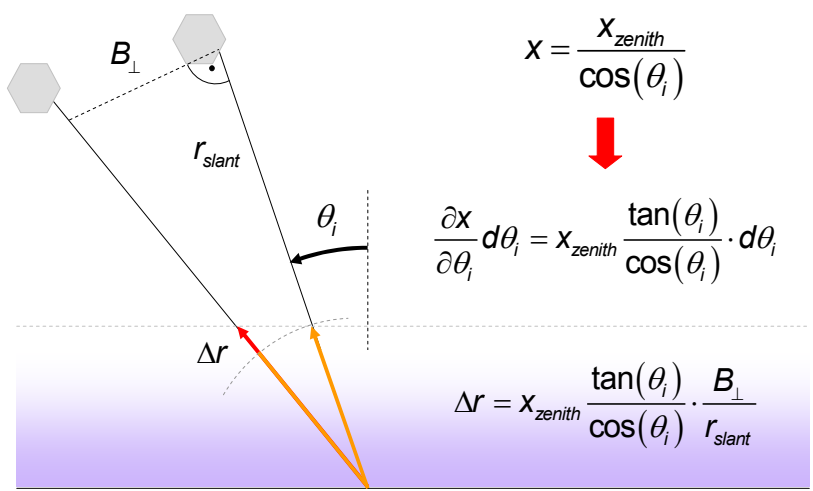

Figure 8: Derivation of differential tropospheric delay.

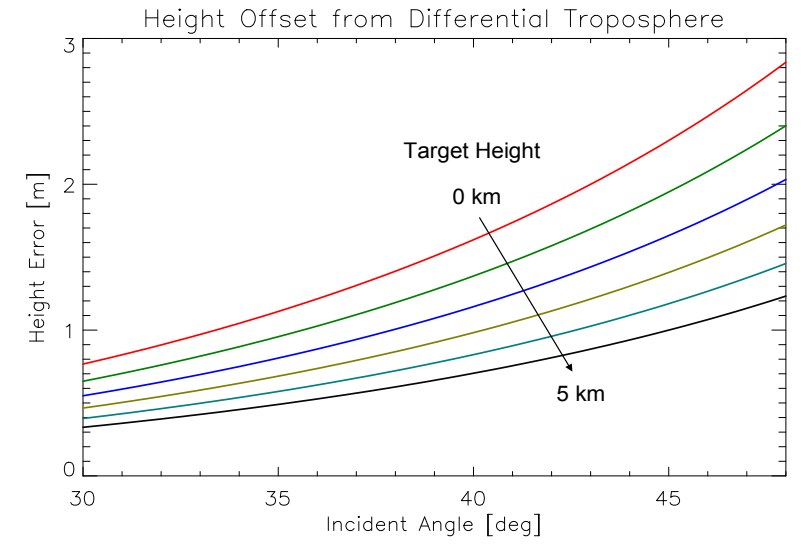

Figure 9: Predicted height offsets for the differential tropospheric delay. The dependence on the incident angle is in good agreement with the observations from Figure 7.

\section{CONCLUSIONS}

Relativistic effects cause notable height offsets in both the radargrammetrically and the interferometrically derived TanDEM-X DEMs. These errors led to a considerable confusion when the first bistatic TanDEM-X DEMs were systematically evaluated. Examples were the almost random distribution of the interferometric phase due to its dependency on the along-track baseline and the puzzling sign flip and latitude dependency of the radargrammetric shifts. Both effects can now be explained by using the theory of special relativity. Differential tropospheric delays caused another unexpected height offset that systematically varied with the incident angle. Appropriate corrections for both effects have in the meantime been implemented in the operational TanDEM-X processor [6].

\section{ACKNOWLEDGMENT}

We thank the TanDEM-X ITP team for helpful discussions and the provision of the radargrammetric and interferometric offsets. The TanDEM-X project is partly funded by the German Federal Ministry for Economics and Technology (Förderkennzeichen 50 EE 1035).

\section{REFERENCES}

[1] G Krieger, A Moreira, H Fiedler, I Hajnsek, M Werner, M Younis, M Zink. TanDEM-X: A Satellite Formation for High Resolution SAR Interferometry. IEEE Trans. Geosc. Remote Sens., vol. 45, pp. 3317-3341, 2007.

[2] J Hueso González, J Walter Antony, M Bachmann, G Krieger, M Zink, D Schrank, M Schwerdt, Bistatic System and Baseline Calibration in TanDEM-X to Ensure the Global Digital Elevation Model Quality, to appear in ISPRS Journal of Photogrammetry and Remote Sensing.

[3] M Rodriguez, unpublished (see 3rd TanDEM-X science meeting: http://www.dlr.de/Portaldata/32/Resources/dokumente/tdmx/sciencemeetin g3/08-Interferometric_Performance_Handout_small.pdf).

[4] A Einstein. Zur Elektrodynamik bewegter Körper. Annalen der Physik und Chemie, vol. 17, pp. 891-921, 1905.

[5] C Misner, K Thorne, J Wheeler. Gravitation. Freeman, 1973.

[6] T Fritz, H Breit, C Rossi, U Balss, M Lachaise, S Duque. Interferometric Processing and Products of the TanDEM-X Mission. IGARSS 2012. 